\title{
THE FEAR CIRCUIT OF THE MOUSE FOREBRAIN: CONNECTIONS BETWEEN THE MEDIODORSAL THALAMUS, FRONTAL CORTICES AND BASOLATERAL AMYGDALA
}

Ferenc Mátyás ${ }^{1}$, JoonHyuk Lee ${ }^{2,3}$, Hee-Sup Shin ${ }^{2,3}$ and László Acsády ${ }^{1}$

${ }^{1}$ Laboratory of Thalamus Research, Institute of Experimental Medicine - Hungarian Academy of Sciences, Budapest H-1083, Szigony u 43. Hungary

${ }^{2}$ Center for Cognition and Sociality, Institute for Basic Science (IBS), Daejeon 305-811, Republic of Korea

${ }^{3}$ Department of Bio and Brain Engineering, Korea Advanced Institute of Science and Technology (KAIST), Daejeon 305-701, Republic of Korea

- Corresponding authors: László Acsády and Ferenc Mátyás

Laboratory of Thalamus Research

Institute of Experimental Medicine, Hungarian Academy of Sciences

Budapest, Szigony u 43 H-1083 Hungary

Email: acsady@koki.hu, matyasf@koki.hu Fax: +36-1210-9413

Running title: Connections of amygdala, prefrontal cortex and thalamus

Total number of pages: 35

Total number of figures: 12

Total number of words in the manuscript: 9288

Total number of words in the Abstract: 250

Total number of words in the Introduction: 554

Keywords: amygdala, thalamocortical network, prefrontal cortex, connectivity, tracing 


\section{ABSTRACT}

A large forebrain circuit, including the thalamus, amygdala and frontal cortical regions, is responsible for the establishment and extinction of fear-related memories. Understanding interactions among these three regions is critical to decipher the basic mechanisms of fear. With the advancement of molecular- and optogenetics, mouse has become the main species to study fear-related behaviours. However, the basic connectivity pattern of the forebrain circuits involved in processing fear has not been described in this species. In this study we mapped the connectivity between three key nodes of the circuit, the basolateral nucleus of amygdala (BLA), the mediodorsal nucleus of the thalamus (MD) and the medial prefrontal cortex, which were shown to have closed triangular connectivity in rats. In contrast to rat, we found no evidence for this closed loop in mouse. There was no major input from BLA to MD and little overlap between medial prefrontal regions connected with both BLA and MD. The common nodes in the frontal cortex, which displayed reciprocal connection with both BLA and MD were the agranular insular cortex and the border zone of cingulate and M2 cortex. In addition BLA can indirectly affect MD via the orbital cortex. We attribute the difference between our results and earlier rat studies to methodological problems rather than to genuine species difference. Our data demonstrate that BLA and MD communicate via cortical sectors whose roles in fear-related behaviour have not been extensively studied. In general, our study provides the morphological framework for the studies of murine fear related behaviours. 


\section{INTRODUCTION}

A significant, adaptive behavioural response of almost all complex organisms is fear. Fear involves the detection of a stimulus that threatens the integrity of the perceiver, the learning of the fearful context, the association of the fearful situation with previously learned stimuli and finally an adaptive behavioural response. Complex cognitive functions require the coordinated activity of several interconnected brain centres. According to our current knowledge, the key forebrain network underlying fear-related behaviours involves the amygdala together with the thalamic and cortical regions in direct connections with it. Indeed, the role of these areas in different aspects of fear behaviour is well established (Sah et al., 2003; Pape \& Pare, 2010; Johansen et al., 2012; Kim et al., 2012; Lee et al., 2012; Marek et al., 2013). However, almost all information about the connectivity pattern of these three brain regions were obtained in rats (Groenewegen, 1988; McDonald, 1998; Heidbreder \& Groenewegen, 2003), whereas recent advances in molecular biology have made the mouse the preferred species of fear studies. As a consequence, it is necessary to establish the large-scale connectivity pattern of the three regions in this species as well.

Data arising from rat on this circuit have been generated by different laboratories using different tracers, and had variable focus. Publications on this topic are frequently separated by decades, making it exceptionally difficult to derive a coherent picture. In addition, the field has two major problems; nomenclature and boundaries. The exact names of the cortical regions in question is in the focus of hot debate, which intensified recently by the suggestion to alter the names of the key medial prefrontal cortical regions involved in fear to match those of the primate/human literature (Vogt et al., 2004; Paxinos \& Franklin, 2013). Names aside, boundaries represent an even more serious and challenging problem. Most borders of the thalamic and cortical regions in question are poorly defined. For example, there are only a 
few attempts to determine the exact borders of pre- and infralimbic cortices (PrL//IL) using neurochemical markers combined with studying the input/output characteristics (Heidbreder \& Groenewegen, 2003; Van De Werd et al., 2010). With no clear borders defined spreading the markers to neighbouring areas is unavoidable. For example, in the thalamus the mediodorsal (MD) nucleus and the midline nuclei appear to have completely different connectivity pattern (see Results) and a very complex border zone, which needs to be carefully established in order to gain a clear picture. Finally, in order to avoid misinterpretation due to the spread of tracers to neighbouring areas, it is advisable to identify every connection in both directions using retrograde and anterograde tracers, preferably by the same laboratory, which has been performed in only a few cases before.

Thus, in this study we used both antero- and retrograde tracings to map the connectivity of three key regions in the circuit, the basolateral amygdala (BLA), the PrL/IL and MD nuclei, which are known to have rich interconnectivity and a well-established role in fearrelated behaviours (Pape \& Pare, 2010; Lee et al., 2012; Marek et al., 2013). By using strict control on injections sites and bi-directional tracing, our data present more limited, connectivity pattern among the three regions compared to earlier studies in rats, and indicate that MD and BLA interact in cortical regions other than PrL/IL. 


\section{MATERIAL AND METHODS}

In all experiments, we used adult male C57BL/6J mice $(\mathrm{n}=77)$. All animal procedures $\mathrm{w}$ ere approved by the Institute of Experimental Medicine Protection of Research Subjects Com mittee as well as the Food-Safety and Animal-Health Office of the Pest District Government Bureau, which is in line with the European Union regulation of animal experimentations. Mice were entrained to a $12 \mathrm{~h}$ light/dark cycle with food and water available ad libitum.

\section{Anterograde and retrograde tracing}

Single or mixtures of an anterograde tracer, Phaseolus vulgaris leucoagglutinin [(Phal, $2.5 \%$ in $10 \mathrm{mM}$ phosphate buffer (PB), $\mathrm{pH} 8.0$; Vector Laboratories] and one retrograde tracer, either Cholera Toxin B subunit (CTB, 0.5\% diluted in distilled water; List Biological) or Fluorogold (FG, 2\% in $0.1 \mathrm{M}$ cacodylate buffer; Fluorochrome), was iontophoretically injected (7-7 sec on/off duty cycle, $3 \mu \mathrm{A}$, for $7 \mathrm{~min}$ for Phal and CTB and 2-2 sec on/off duty cycle, $2 \mu \mathrm{A}$, for $7 \mathrm{~min}$ for $\mathrm{FG}$ ) into the following brain regions (coordinates relative to Bregma): prelimbic cortex (PrL; AP/L/DV 2.0/0.3/2.0), cingulate cortex (Cg; 1.0-2.0/0.3/1.0) agranular insular cortex (AI; 0.9-1.4/3.0/2.6), basolateral amygdala (BLA; -1.3-1.6/3.4/4.0), mediodorsal thalamus (MD; -1.0-1.5/0.4/3.2) under ketamine $(75 \mathrm{mg} / \mathrm{kg}) /$ xylazine $(5 \mathrm{mg} / \mathrm{kg})$ anaesthesia. After one week, the animals were perfused first with saline (0.9\%) and then with a $4 \%$ paraformaldehyde $(\mathrm{TAAB})$ solution $(100 \mathrm{~mL})$. Then, brains were removed and cut into $50 \mu \mathrm{m}$ thick coronal sections with Leica Vibratome.

\section{Immunocytochemistry}

Sections were intensively washed with phosphate buffer (PB; $0.1 \mathrm{M})$ and then treated with a blocking solution containing $5 \%$ Normal Donkey Serum (NDS; Millipore) and $0.5 \%$ Triton-X (Sigma) for 30 minutes at room temperature. The following primary antibodies diluted in PB containing $0.1 \%$ NDS and $0.1 \%$ Triton-X were used: anti-Phal (rabbit 
1:10000-20000; Vector Laboratories), anti-CTB (goat 1:2-30000; List Biological), anti-FG (rabbit, 1:10000-20000; Fluorochrome), anti-parvalbumin (Parv; rabbit or mouse 1:30005000; Swant) and anti-vesicular glutamate transporter type 2 (vGluT2; mouse or guinea pig 1:2000-5000; Chemicon and SYSY). After primary antibody incubation (1 day at room temperature, or 2-3 days at $4{ }^{\circ} \mathrm{C}$ ), sections were treated with secondary antibodies labelled with either fluorescent dye (Alexa-488, Cy3 or Alexa 647; 1:500, 2 h at RT; Molecular Probes and Jackson) or biotin (1:300, $2 \mathrm{~h}$ at RT; Vector Laboratories). The biotin-treated sections were further incubated with an avidin-biotin complex (ABC, Vector Laboratories; 1:300, $2 \mathrm{~h}$ ) and developed by nickel intensified diaminobenzidine (DABNi; Sigma) as a chromogen. Sections with fluorescent staining were mounted in Vectashield with DAPI (Vector Laboratories) and those with DABNi in DEPEX (Serva).

Photographs were taken using either a Zeiss Axioplan 2 microscope equipped with a DP70 (Olympus) or a Zeiss digital camera, or an Olympus FluoView FV1000 confocal laser scanning system. When necessary, brightness and contrast were adjusted using Adobe Photoshop CS5 (Adobe Systems) applied to whole images only. 


\section{RESULTS}

\section{Boundaries, nomenclature}

For the parcellation of the medial wall of the prefrontal cortex in mice we took the chemoarchitectonic approach of (Van De Werd et al., 2010) in mice as a starting point and combined it with input/output characterization of this region. We compared the boundaries apparent in parvalbumin-immunostained sections to the distribution of amygdalo-cortical fibres, cortico-amygdaloid cells and thalamocortical inputs (Fig. 1).

Based on these markers, two well-defined borders were apparent. A dorsal border was defined by an abrupt change in parvalbumin-immunoreactive cell bodies and fiber staining in the superficial cortical layers (Fig. 1A). Dorsal to this border there is a dense meshwork of parvalbumin-positive fibres and somata which is barely noticeable ventrally. Parvalbumin staining in the deep layers also becomes weaker, but cell bodies are still immunostained. The same dorsal border is defined by a significant and sharp drop in the density of amygdalar input in the superficial cortical layers, and an abrupt shift of this input to the deeper cortical layers (Fig. 1B-C). The position of cortical cells retrogradely labelled from the amygdala also changes. Dorsal to the border, cortico-amygdalar cells are apparent only in superficial layers, whereas ventrally cells are located in deeper layers as well (Fig. 1B,D). Finally, this border roughly coincides with the dorsal margin of a dense meshwork of fibres arising from the midline thalamic nuclei (Fig. 1E) (Hoover \& Vertes, 2007; Li \& Kirouac, 2008).

The ventral border is characterized by the re-emergence of dense parvalbumin staining in the mid-cortical layers (Fig. 1A) and the reappearance of dense amygdalar input which extends from deep to superficial layers in this region (Fig. 1B and C). The amygdalo-cortical cells in deeper layers disappear or shifts back to superficial layers (Fig. 1B, D), whereas the midline input becomes very sparse ventrally to this border (Fig. 1E). 
These borders are apparent through the entire antero-posterior extent of this region and their position is relatively stable, as measured from the dorsal surface of the brain. The dorsal border is around 1.3-1.5 $\mathrm{mm}$ from the apex of the brain surface in fixed brains, whereas the coordinates of the ventral border are $2.0-2.2 \mathrm{~mm}$. Calculating for $10 \%$ shrinkage due to fixation, these values become 1.4-1.7 $\mathrm{mm}$ and 2.2-2.4 $\mathrm{mm}$ in the living brain.

Based on the earlier data (Van De Werd et al., 2010) and using the old nomenclature, we term the region between the dorsal and ventral border (i.e. receiving no superficial amygdalar input but heavy midline thalamic input) prelimbic cortex (PrL) and the region ventral to the ventral border (i.e. contacted by heavy amygdalar but few or no midline fibres) infralimbic cortex (IL). We are, however, aware of the controversy, which argues that these two names are the misnomer of the rodent literature and the suggestion to replace these names with the Brodmann numbers (Vogt et al., 2004). Still, presently we are hesitant to use these new names here, since in the new edition of the Paxinos atlas (Paxinos \& Franklin, 2013) the borders for regions 24, 25 and 32 are not entirely compatible with ours. Thus, for the sake of the present study we will use the old terms, noting that we are ready to switch them to the new names when these issues are settled.

Regarding other cortical regions, we refer to the cortical area dorsal to PrL as cingulate cortex. The lateral border of cingulate cortex roughly coincides with the lateral spread of cortico-amygdalar cells. We term the cortical region lateral to cingulate (and having many different names: whisker motor, medial agranular cortex etc.) M2 cortex, in accord with Paxinos and Franklin (Paxinos \& Franklin, 2013). We refer to the agranular insular cortex as insular cortex here for brevity.

Regarding the borders of MD, from the point of view of this study the most important is to delineate MD and the midline nuclei, especially in the rostral thalamic regions where midline nuclei have a lateral wing and the paratenial nucleus appears in the position of MD. 
We found that the most convenient marker for this region was vesicular glutamate transporter 2 (vGLUT2) (Fig. 2). Since this marker labels subcortical inputs (Fremeau Jr. et al., 2001; Herzog et al., 2001; Lavallee et al., 2005; Graziano et al., 2008) and midline nuclei are known to receive strong glutamatergic afferents from the brainstem (Krout et al., 2002), which is weaker in MD (Groenewegen, 1988), we found this marker especially suitable to label the medial border of MD along most of its antero-posterior extent (Fig. 2). However, in the rostral pole of MD where MD abruptly changes in size, the pronounced difference the difference in the levels of vGLUT2 immunostaining between MD and midline nuclei is less visible (Fig. 2A) (Fig. 2A). We refer to this zone, between Br. $-0.8 \mathrm{~mm}$ and $-1.1 \mathrm{~mm}$ as a transition zone since there are several indications that it contains thalamocortical neurons with heterogeneous (MD and midline) nuclear origin. It is important to distinguish this zone from the medial MD (MDm) as defined by Paxinos and Franklin (Paxinos \& Franklin, 2013). $\mathrm{MDm}$ is located caudally to the transition zone and is present throughout the entire antroposterior extent of the nucleus whereas the transition zone tapers off in the rostral part of the nucleus (Fig 2B).

In order to map the connectivity between MD, BLA and the various cortical regions altogether 89 tracer injections were made, some of them in combination. Retrograde tracers were injected in 21 cases in the thalamus. Cortical regions were injected with anterograde tracers $(n=20)$ and retrograde tracers $(n=32)$. Anterograde $(n=6)$ and retrograde $(n=10)$ tracers were also injected in the amygdala.

\section{Connectivity between thalamus and neocortex}

In order to map the cortical regions projecting to $\mathrm{MD}$, we injected retrograde tracers (Fluorogold or Cholera Toxin) into MD ( $\mathrm{n}=21$ all cases) and examined the distribution of 
corticothalamic cells (Fig. 3). The tracer was entirely confined within the boundaries of MD as defined by vGLUT2 immunostaining in 4 cases (Fig. 3D). The injection site encroached the centrolateral and/or the parafascicular nucleus (laterally and caudally) in 6 cases, whereas it reached the midline nuclei and/or the anterior transition zone in 11 cases. Separate injections targeting the centrolateral/parafascicular nuclei and the midline nuclei were also performed.

In all cases, when the tracer injection was confined to MD, a consistent pattern of cortical labelling emerged. Regardless of the exact antero-posterior location of the injection site, two major cortical cell streams were labelled following each MD injection, a dorsal and a lateral stream (Fig. 3A-C). In the dorsal stream the vast majority of the neurons were located in the M2 cortex and along the border of M2 and cingulate cortex (Fig. 3C). Only few scattered cells were found in the rostral PrL and IL where they merge with the orbital cortices and the borders in deep layers are difficult to define (Fig. 3B, E-F). Similarly, no neurons were observed in M1 cortex lateral to M2. In the lateral stream, the neurons were confined to a narrow region within the insular cortex (Fig. 3C). In the anterior portion of the frontal cortex the two streams converged and a large population of neurons appeared, encompassing the M2, and ventral and lateral orbital cortices (Fig. 3A). Most labelled cells were confined to layer 6, with layer 5 containing scattered, individual neurons.

When the injection site included the centrolateral or the parafascicular nuclei, in addition to the pattern described above, a significant number of retrogradely labelled cells appeared in the M1 motor cortex. When the injection site extended medially and included the midline nuclei, or involved the rostral, transition zone numerous labelled cells appeared in the PrL and IL. Targeted injection to midline and intralaminar nuclei confirmed these observations (data not shown). 
In order to confirm the observations obtained by the retrograde labelling and to map the distribution of corticothalamic fibres within the MD originating from the cortex, we injected anterograde tracer into cingulate/M2 cortex $(\mathrm{n}=10), \operatorname{PrL} / \mathrm{IL}$ cortex $(\mathrm{n}=6)$ and insular cortex $(n=4)$ (Fig. 4). Cortical fibres originating from different cortical regions were largely confined to distinct sectors within the MD. Fibres from the cingulate/M2 region cortex extensively innervated the intralaminar nuclei and the lateral and dorsal part of MD (Fig. 4A-C) as shown before (Kim et al., 2012). Moving the injection site from the medial to the lateral part within this zone resulted in a progressively more ventral innervation zone within MD. Moving the injection site ventrally from cingulate to PrL resulted in a sudden shift in the innervation pattern. Instead of the lateral margin of MD most of the fibres were localized in the rostral and medial transition zone between midline nuclei and MD (Fig. 4D-F). Injections into the insular cortex, in contrast, labelled a compact band of fibres in the transition zone as well as in medial part of MD along the whole antero-posterior extent of the nucleus (Fig. 4G-I).

The reciprocal, thalamocortical connections were examined by injecting retrograde tracers to cingulate $(n=11), \operatorname{PrL} / \mathrm{IL}(\mathrm{n}=13)$ and insular cortices $(\mathrm{n}=8)$ (Fig. 5). In all three cases the pathways displayed precise reciprocity i.e. the position of thalamocortical cells coincided with the respective corticothalamic fibres. In details, the injection of the tracer into the cingulate cortex resulted in retrogradely labelled cells in the dorsal and lateral aspect of MD (Fig. 5A-C), as shown before (Kim et al., 2012). After PrL/IL injections the vast majority of retrogradely labelled cells were confined to the transition zone along the rostral midline/MD border (Fig. 5D-F) and the bulk of MD was free of retrogradely labelled cells. Finally, following the labelling of insular cortex, many neurons appeared in the medial portion of MD outside the midline nuclei (Fig. 5G-I).

\section{Connectivity between the amygdala and the thalamus}


In order to map the amygdalo-thalamic pathway we used the same animals injected by retrograde tracers in the MD (see above). None of the retrograde tracers used revealed a substantial number of retrogradely labelled cells in the BLA, lateral amygdala (LA) or central nuclei (Fig. 6). In all animals with thalamic injections, the number of neurons in these nuclei never exceeded 1-2/section, and in many sections no neuron was found at all. However, higher number of neurons (occasionally 5-8 cell/sections) were consistently observed in a small nucleus located lateral to BLA, the ventral endopiriform nucleus (vEN; Fig. 6A,B). Finally, a few neurons were also labelled in medial and caudal amygdalar nuclei (up to 10/sections) (Fig. 6C).

In order to check the results obtained by the retrograde tracing, anterograde tracers were injected into the BLA (n=6) (Fig. 7). When the injection site avoided the lateral margin of BLA no thalamic fibres were revealed (Fig. 7A-C). When the injection sites involved the ventral endopiriform nucleus together with the lateral part of BLA, scattered fibres with largesized axon terminals were observed in the medial part of MD adjacent to the midline nuclei (Fig. 7D-F). In order to positively identify the origin of these fibres we examined their vesicular glutamate transporter (vGLUT) content. Since BLA contains vGLUT1-positive neurons whereas in the ventral endopiriform nucleus vGLUT2-containing cells are abundant (Hur \& Zaborszky, 2005), the vGLUT content can confirm the origin of the fibres. Indeed, as suggested by the localization of the tracer injection, the thalamic fibres anterogradely labelled by tracer injection involving the BLA and the ventral endopiriform nucleus were negative for vGLUT1 (Fig. 7G) but positive for vGLUT2 (Fig. 7H). These data demonstrate the lack of significant, direct connection between BLA and MD.

Finally, in order to map the location of thalamic neurons projecting to the amygdala, retrograde tracers were injected into the amygdala (BLA/LA, n=10; Fig. 8A). Thalamic neurons located in the MD were not found following any injections, however large number of 
thalamic neurons was visualized in the midline nuclei (Fig. 8B-D) as describe in rat before (Ottersen \& Ben-Ari, 1979; Su \& Bentivoglio, 1990; Li \& Kirouac, 2008) and confirmed recently in mice with anterograde tracing (Dobi et al., 2013). Substantial numbers of retrogradely labelled cells was also found in the posterior intralaminar thalamic/ suprageniculate nuclei (data not shown) in those cases in which the retrograde tracer also penetrated the LA as described before (Doron, 1999).

\section{Connectivity between the amygdala and the cortex}

The same retrograde injections were used to localize cortico-amygdalar neurons as above to locate thalamo-amygdalar cells (Fig. 9). Following the injection of retrograde tracer to the BLA a dense and continuous band of labelled neurons appeared in the medial prefrontal cortex from the IL through PrL and extending to the cingulate cortex (Fig. 9A-E). In the cingulate cortex, very few or no retrogradely labelled cells were found posterior to the Bregma $+1.0 \mathrm{~mm}$ coronal level (Fig. 9A-D). A second band of labelled cells appeared laterally in the insular cortex (Fig. 9A-C and F). Cortico-amygdalar cell were located mainly in layer 2/3 but PrL and IL contained labelled cells in deeper layers as well.

Injections of the anterograde tracers to cingulate $(n=10), \operatorname{PrL} / \mathrm{IL}(\mathrm{n}=6)$ and insular $(\mathrm{n}=4)$ cortices confirmed the results obtained by retrograde tracing and revealed a dense corticoamygdalar input localized mainly to BLA (Fig. 10). Injections targeted to the cingulate cortex resulted in significant fibre labelling only when the injections sites were located rostral the Bregma $+1.0 \mathrm{~mm}$. Within the BLA cingulate fibres were more dense in the dorsal than ventral part of the nucleus whereas PrL inputs were more evenly distributed (Fig. 10A-D; see also in Cho et al., 2013). In addition, PrL axons also reached the ventral part of LA. Insular input was less dense in the BLA and also targeted the lateral nucleus (Fig. 10E and F). The central amydaloid nuclei received only negligible cortical inputs of any cortical sources. Following 
insular and cingulate/M2 injections labelled fibres appeared simultaneously in both MD (Fig. 4) and BLA (Fig. 10) demonstrating co-innervation of thalamus and amygdala from the same cortical region.

Next we characterized the reciprocal amygdalo-cortical pathways using both anterograde and retrograde tracings using the same animals as for corticothalamic and thalamocortical experiments (see above). Following the injection of retrograde tracers to cingulate (Fig. 10G and H) and PrL/IL cortices (Fig. 10I and J), a large number of neurons was labelled in BLA, whereas insular injections resulted in fewer neurons in BLA and labelled cells also appeared in LA (Fig. 10K and L). The location of the amygdalo-cortical cells displayed close correspondence to the distribution of the respective cortico-amygdalar fibres. The central amygdaloid nuclei were virtually free of neurons projecting to any cortical regions investigated. Labelled cells simultaneously appeared in both MD (Fig. 5) and BLA (Fig. 10) following insular and combined cingulate/M2 injections, demonstrating coinnervation of these cortical regions by amygdala and thalamus.

Finally, in order to identify the amygdalo-cortical fibres, anterograde tracers were injected to the amygdala (Fig. 11). All three cortical regions (PrL/IL, cingulate, insular) containing amygdala-projecting neurons also received amygdalar inputs, demonstrating the reciprocity of these pathways. However, the BLA innervated much larger cortical regions than it received cortical input from, indicating that many amygdalo-cortical pathways are unidirectional and not reciprocated. Amygdalo-cortical fibres could be observed to travel caudally along the cingulate bundle reaching the retrosplenial cortices. Laterally, amygdalar fibres crossed the M2 regions and extended to M1 cortex (Fig. 11A and B). In the rostral pole, besides PrL/IL and cingulate labelling, a dense meshwork of fibres was observed in the lateral and ventral orbital cortices as well (Fig. 11A and B). The amygdalo-cortical pathway targeted mainly the same layer which contained the cortico-amygdalar cells (layer 2/3) but extended to 
deeper layers in IL and the M2 areas (Fig. 11A, B, C and E). These data show that major cortical regions which innervate MD (M2, ventral and lateral orbital cortices) receive input from BLA.

\section{$\underline{\text { Cortico-cortical connections }}$}

Injections to the cingulate, insular and PrL/IL cortical areas allowed us to investigate the interconnectivity of these cortical regions (data not shown). Briefly, pronounced bidirectional connection was found between insular and PrL/IL cortices. However, cingulate cortex, received only sparse input from insular cortex, restricted to the superficial layers in along the medial wall of cingulate, never extending to the cingulate/M2 border. Cingulate cortex also had surprisingly weak connectivity with PrL/IL cortices. Following cingulate injections of retrograde tracers only a specific caudal region of the PrL/IL cortices contained labelled cells. 


\section{DISCUSSION}

In the present study we found limited interconnectivity between BLA, MD and PrL/IL in mice compared earlier rat studies (Fig. 12). As we discuss below, this can be largely attributed to methodological rather than to species differences. According to our data, BLA and the main body of MD have no direct connection and PrL/IL is not a common node between them. However, BLA and MD can interact via mutual reciprocal connections with the insular cortex and the cingulate/M2 border region. In addition, amygdalar fibres innervate ventral and lateral orbital cortices which project to MD. Since the role of these cortical regions in fear-related behaviours is presently less clear, our data highlights the importance of studying these regions in the future.

\section{Connectivity between MD and cortical region}

According to our data, the main body of MD has reciprocal connection with insular, orbital and cingulate/M2 regions but not with PrL/IL (Fig. 3-5). Connectivity with PrL/IL is restricted to a rostral/medial transition zone between MD and midline nuclei. These data in mice are broadly consistent with earlier results obtained in rat (for a review see Heidbreder \& Groenewegen, 2003) with important limitations.

The distribution of cortical cells projecting to MD in our study is more restricted (Fig. 3) compared to earlier studies in rats using large MD injections (Groenewegen, 1988; Gabbott et al., 2005) but similar to those using small injections (Cornwall \& Phillipson, 1988; Ray et $a l .$, 1992). This is probably due to the fact that we systematically excluded all injection sites which encroached upon neighbouring nuclei.

The fact that PrL/IL is connected only with the rostral/medial transition zone of MD is supported by several earlier observations in rat. Following retrograde tracing from the 
thalamus Ray et al., (Ray et al., 1992) described PrL/IL cortical cell labelling only after medial MD injection, which involved the midline nuclei, but not following more lateral injections (compare their Fig. 2 with 3 and 4). Similarly, following cortical anterograde tracing PrL/IL fibres were found to innervate only the rostral MD, which we identify here as a transition zone but not to the main body of MD (Hurley et al., 1991; Vertes, 2004) a result which we also confirmed here in mice (Fig. 4D-F).

Tracing the localization of thalamocortical cells projecting to limbic cortices in this study gives further support to the preferential connection between the rostral/medial transition zone of MD and PrL/IL (Fig. 5D-F). These results, however, are at variance with earlier data (Ray \& Price, 1992), which describe considerable MD input to PrL/IL using retrograde cortical injections. We think, however, in this rat that study the diffusion of the tracer to dorsal cingulate regions cannot be excluded and as we show here thalamic cells projecting to cingulate cortex can be found in the main body of MD unlike PrL/IL projecting cells.

Our findings are consistent with the data available on the on-line mouse atlas of mouse neuronal pathways (Allen Brain Atlas). In case 168300739 of this atlas the injection of MD with anterograde virus results in fibres innervating only cingulate cortex but not PrL. In contrast, the more rostral thalamic injection targeting the region we term transition zone (case 168301446) essentially provides a complementary pattern i.e. afferents are evident in PrL but not in cingulate cortex. However, since Allen Brain Atlas does not use the neurochemical borders we defined here for PrL and MD, their interpretation of the data is different.

It is important to indicate a major caveat of thalamic tracing studies here. Nuclear borders are not definite lines in the thalamus but always represent a gradient with neurons extending dendrites into neighbouring nuclei, showing transient electrophysiological features 
(Slézia et al., 2011) and/or scattered neurons embedded in the matrix of one nucleus bearing the properties of another (Jones, 1998). In addition, the diffusion of tracers cannot be completely controlled using mass injections. In our case the anterograde and retrograde tracings from the cortex together indicate that there is a gradient between heavy PrL/IL connectivity (midline nuclei) to no connectivity (MD) with a transient along the borders. The question can be more precisely determined in the future only by labelling individual cortical and thalamic cells, preferentially using appropriate, nucleus specific Cre-lines.

Following retrograde tracer injections to MD two other studies (Gabbott et al., 2005) and (Groenewegen, 1988) report an extensive cortical labelling, including primary motor cortices as well. In both of these cases, large injections were made that appear to include the intralaminar nuclei as well to some extent (see Fig. 8-15 of Groenewegen, 1988 and Fig. 8D of Gabbott et al., 2005). In our study we found M1 neuronal only labelling when the injections sites reached the intralaminar nuclei. This is consistent with earlier rat data (Alloway et al., 2009) using anterograde tracing of the motor cortex-thalamus pathway and reporting intralaminar input when the lateral (M1 or forepaw motor cortex) is injected but the appearance of MD fibres when the more medial motor cortex (i.e. M2 or whisker motor cortex) is targeted. Tracing the reciprocal, thalamocortical pathway, Wang and Shyu (Wang \& Shyu, 2004) found thalamic fibres in M1 only when the tracer was injected into the intralaminar nuclei (CL), and not when MD was targeted. Based on these findings significant motor cortical connections of MD may be excluded.

There is less controversy within the literature regarding the connectivity of MD with the M2/cingulate regions, the insular cortex and the lateral and ventral orbital cortices (Condé et $a l .$, 1995). This pattern is emerging in every study reviewed above. It is important to note here that $\operatorname{PrL}$ and insular cortices have at least partially overlapping termination zone in MD (Fig. $4 \mathrm{E}, \mathrm{H})$ and are also connected with each other. In contrast, the cingulate cortex targets a 
distinct sector within MD (Fig. 4B,C) and is only weakly connected with PrL and insular regions. This suggests the presence of parallel cortical functional loops within the MD.

In summary, from the point of view of the present work, it is clear that with the possible exception of a border region with the midline nuclei the bulk of MD does not receive PrL/IL input and does not have a major projection to these areas. This certainly restricts the cortical regions where MD and amygdalar information can directly interact (see below).

\section{Connectivity between the amygdala and thalamus}

Earlier studies in rats indicated a prominent input from the amygdaloid complex to the medial portion of MD (Groenewegen, 1988; Ray et al., 1992; Reardon \& Mitrofanis, 2000). These fibres were very few in number but bore large terminals and established multiple contacts on the proximal dendrites of relay cells (Kuroda \& Price, 1991), thus they were considered as a "driver" input of these neurons (Sherman \& Guillery, 1998). Considering the importance of the amygdala in fear-related behaviours, this indicated a major involvement of medial MD in processing fear signals to the frontal cortex.

In the present study, however, no major input was found between BLA and MD. Rather, our data indicate that a small nuclei lateral to the BLA, the ventral endopiriform nucleus (VEn), is responsible for the bulk of the large terminals observed in medial MD. The position and type of fibres in MD described in these earlier studies exactly match our observations of lateral BLA injections, which included VEn. Our conclusion is supported by our anterograde and retrograde tracings together with the distinctive neurochemical marker content (vGLUT2) of these fibres. The data are broadly consistent with an earlier retrograde study (Groenewegen, 1988) describing very few retrogradely labelled cells in the BLA, and extensive input from the ventral endopiriform nucleus. We think that the difference between our study and the earlier anterograde tracing studies does not arise because of the different 
species used here (mouse rather than rat), but is rather due to the involvement of the small nucleus VEn, along the lateral aspect of BLA, upon large anterograde injections made in the amygdala. Indeed in these studies (Groenewegen, 1988; Ray \& Price, 1992) the involvement of VEn cannot be excluded since no systematic analysis of medial vs. lateral BLA injections was performed. The functional relevance of the VEn-BLA projection is presently unclear since the firing activity of VEn during fear-related behaviours and their detailed connectivity with BLA is unknown.

Regarding the reciprocal thalamo-amygdalar input our data replicate the condition described in rat (Ottersen \& Ben-Ari, 1979; LeDoux et al., 1990; Su \& Bentivoglio, 1990; Li \& Kirouac, 2008) and recently confirmed in mice (Dobi et al., 2013), that there is a lack of input from MD and a major input arising from both the midline and the posterior intralaminar nuclei.

In summary, we conclude that in mice, direct connection between MD and amygdala does not play a major role in processing fear-related signals. We suspect that the information conveyed by MD and amygdala is rather integrated by well-defined cortical regions (see below).

\section{Connectivity between the amygdala and prefrontal cortex}

In general, the connectivity of prefrontal cortex and amygdala in mice is compatible with earlier data in rats (for a review see McDonald, 1998; Heidbreder \& Groenewegen, 2003). In particular, the distribution of cortico-amygdaloid cells in PrL/IL and cingulate cortices is comparable with data obtained in rats (Krettek \& Price, 1977; Ottersen, 1982; Cassell \& Wright, 1986). We have to add, however, that there is a sharp caudal limit of cortico-amygdaloid cells in the cingulate cortex (at around Bregma $+1.0 \mathrm{~mm}$ coronal level), which was demonstrated both by anterograde and retrograde tracing here but not explicitly 
stated earlier. The distribution of cortical fibres in the BLA arising in cingulate and insular areas in rat (Mcdonald et al., 1996) precisely matches our observations in mouse. Recent data in mice demonstrate significant input to BLA in mice from PrL/IL (Cho et al., 2013).

Considering the reciprocal pathway, our data are also entirely consistent with the rat data. Using retrograde tracing from the prefrontal cortex, the projection of BLA to the medial frontal and insular cortices has been well established (Sripanidkulchai et al., 1984; McDonald, 1991; Shinonaga et al., 1994). Similar to our results, Conde and colleagues (Condé et al., 1995) found retrogradely labelled cells in the BLA only when the cingulate and more medial cortex was injected, and not when lateral motor cortices were labelled. Regarding the anterograde tracing of the amydalocortical fibres we are aware of just a single study in rats (Kita \& Kitai, 1990). This study demonstrated the innervation of insular, cingular and IL areas and the spread of amygdaloid fibres to the more lateral motor cortices and the orbital cortices, in concert with our data. These data together with ours suggest that the BLA sends information to larger cortical areas than it receives input from in both species and welldefined cortical regions have reciprocal connectivity with both BLA and MD.

\section{Functional implications}

The role of BLA and PL/IL in fear conditioning is very well established and supported by many studies (for a review see Pape \& Pare, 2010; Johansen et al., 2012; Marek et al., 2013). In addition, MD has also been shown to be involved in various aspects of fear behaviour (Herry \& Garcia, 2002; Jeon et al., 2010; Lee et al., 2012). These data were nicely supported by the close, triangular connectivity between the three forebrain regions as suggested by the earlier tracing studies in rats (Fig. 12A). According to these data, MD could serve as an indirect link between BLA and PrL/IL and information processed by MD and BLA could have been integrated in PrL/IL. These hypotheses gain little support from our 
data, showing the paucity of BLA-MD and MD-PrL/IL connections (Fig. 12B). So the problem arises, how and where fear related signals of MD and BLA are integrated or in general, how thalamic and amygdalar information is combined. We propose two scenarios here. One involves the midline nuclei and the midline-MD transition zone since it is connected to both BLA and PrL/IL. The other includes two well-defined cortical regions (M2/cingulate border region and insular cortex) which have bidirectional connection with both BLA and MD could serve as links between the two regions (Fig. 12C; and also see Fig. 3C and Fig. 9B,C). It should be kept in mind, however, that different layers project to thalamic and amygdalar targets (Fig. 3 and 9), thus integration of the signals arising from these sources likely requires intracortical interactions and not direct co-modulation of the two targets by the same cortical neurons. Cingulate and insular inputs are clearly segregated in the MD (Fig. 4) and to some extent in BLA (Fig. 10). These two cortical regions have little interconnectivity suggesting that the two cortical regions are involved in parallel circuits and thus, may subserve different aspects of fear behaviour. Finally, the unusual, unidirectional influence from BLA to orbital cortices which are themselves connected to MD indicates a one way BLA-MD influence within the circuit (Fig. 12C). In this case amygdalar fibres overlap with corticothalamic cells (compare Fig. 3 and Fig. 11), thus, direct interaction may take place. We should also point out, however, that the role of the cortical regions which has the potential to link BLA and MD activity is little understood. Furthermore, in general, the role of ascending amygdalar input and thalamocortical interactions in fear behaviour certainly necessitate more investigation in this area. 


\section{Acknowledgements}

This work was supported by Institute for Basic Science (IBS). and the Korean-Hungarian

Joint Laboratory Program, the National Honor Scientist Program of Korea, Hungarian Scientific Research Fund (OTKA T75676) the National Office for Research and Technology (NKTH-ANR, Neurogen) and Hungarian Brain Research Program - Grant No. KTIA_13_NAP-A-I/1. F.M. is János Bolyai Research fellow. The excellent technical help of Krisztina Faddi is gratefully acknowledged. The authors declare no conflict of interest.

\section{Abbreviations}

$\mathrm{AI}$ - agranular insular cortex

BLA - basolateral nucleus of amygdala, anterior part

$\mathrm{Cg}$ - cingulate cortex

$\mathrm{CL}$ - centrolateral thalamic nucleus

$\mathrm{CM}$ - centromedial thalamic nucleus

CTB - Cholera Toxin-B subunit

FG - Fluorogold

$\mathrm{Hb}$ - habenula

IL - infralimbic cortex

IMD - intermediodorsal thalamic nucleus

LA - lateral amygdala

M1 - primary motor cortex

M2 - secondary motor cortex

MD - mediodorsal nucleus of thalamus

MeA - medial amygdala

Parv - parvalbumin 
$\mathrm{PC}$ - paracentral thalamic nucleus

Phal - Phaseolus vulgaris leucoagglutinin

PrL - prelimbic cortex

$\mathrm{PV}$ - paraventricularis thalamic nucleus

VEn - ventral endopiriform nucleus

$\mathrm{VO} / \mathrm{LO}$ - ventral and lateral orbital cortices

\section{REFERENCES}

Alloway, K.D., Smith, J.B., Beauchemin, K.J., \& Olson, M.L. (2009) Bilateral projections from rat MI whisker cortex to the neostriatum, thalamus, and claustrum: forebrain circuits for modulating whisking behavior. J. Comp. Neurol., 515, 548-564.

Cassell, M.D. \& Wright, D.J. (1986) Topography of projections from the medial prefrontal cortex to the amygdala in the rat. Brain Res. Bull., 17, 321-333.

Cho, J.-H., Deisseroth, K., \& Bolshakov, V.Y. (2013) Synaptic Encoding of Fear Extinction in mPFC-amygdala Circuits. Neuron,.

Condé, F., Maire-Lepoivre, E., Audinat, E., \& Crépel, F. (1995) Afferent connections of the medial frontal cortex of the rat. II. Cortical and subcortical afferents. J. Comp. Neurol., $352,567-593$.

Cornwall, J. \& Phillipson, O.T. (1988) Afferent projections to the dorsal thalamus of the rat as shown by retrograde lectin transport--I. The mediodorsal nucleus. Neuroscience, 24 , 1035-1049.

Dobi, A., Sartori, S.B., Busti, D., Van der Putten, H., Singewald, N., Shigemoto, R., \& Ferraguti, F. (2013) Neural substrates for the distinct effects of presynaptic group III metabotropic glutamate receptors on extinction of contextual fear conditioning in mice. Neuropharmacology, 66, 274-289.

Doron, N.N. (1999) Organization of Projections to the Lateral Amygdala From Auditory and Visual. J. Comp. Neurol., 412, 383-409.

Fremeau Jr., R.T., Troyer, M.D., Pahner, I., Nygaard, G.O., Tran, C.H., Reimer, R.J., Bellocchio, E.E., Fortin, D., Storm-Mathisen, J., \& Edwards, R.H. (2001) The expression of vesicular glutamate transporters defines two classes of excitatory synapse. Neuron, 31 , 247-60. 
Gabbott, P.L. a, Warner, T. a, Jays, P.R.L., Salway, P., \& Busby, S.J. (2005) Prefrontal cortex in the rat: projections to subcortical autonomic, motor, and limbic centers. J. Comp. Neurol., 492, 145-177.

Graziano, A., Liu, X.-B., Murray, K.D., \& Jones, E.G. (2008) Vesicular glutamate transporters define two sets of glutamatergic afferents to the somatosensory thalamus and two thalamocortical projections in the mouse. J. Comp. Neurol., 507, 1258-1276.

Groenewegen, H.J. (1988) Organization of the afferent connections of the mediodorsal thalamic nucleus in the rat, related to the mediodorsal-prefrontal topography. Neuroscience, 24, 379-431.

Heidbreder, C.A. \& Groenewegen, H.J. (2003) The medial prefrontal cortex in the rat: evidence for a dorso-ventral distinction based upon functional and anatomical characteristics. Neurosci. Biobehav. Rev., 27, 555-579.

Herry, C. \& Garcia, R. (2002) Prefrontal cortex long-term potentiation, but not long-term depression, is associated with the maintenance of extinction of learned fear in mice. $J$. Neurosci., 22, 577-583.

Herzog, E., Bellenchi, G.C., Gras, C., Bernard, V., Ravassard, P., Bedet, C., Gasnier, B., Giros, B., \& El Mestikawy, S. (2001) The existence of a second vesicular glutamate transporter specifies subpopulations of glutamatergic neurons. J Neurosci, 21, RC181.

Hoover, W.B. \& Vertes, R.P. (2007) Anatomical analysis of afferent projections to the medial prefrontal cortex in the rat. Brain Struct. Funct., 212, 149-179.

Hur, E.E. \& Zaborszky, L. (2005) Vglut2 afferents to the medial prefrontal and primary somatosensory cortices: a combined retrograde tracing in situ hybridization study [corrected]. J. Comp. Neurol., 483, 351-373.

Hurley, K.M., Herbert, H., Moga, M.M., \& Saper, C.B. (1991) Efferent projections of the infralimbic cortex of the rat. J. Comp. Neurol., 308, 249-276.

Jeon, D., Kim, S., Chetana, M., Jo, D., Ruley, H.E., Lin, S.-Y., Rabah, D., Kinet, J.-P., \& Shin, H.-S. (2010) Observational fear learning involves affective pain system and Cav1.2 Ca2+ channels in ACC. Nat. Neurosci., 13, 482-488.

Johansen, J., Cain, C., Ostroff, L., \& LeDoux, J. (2012) MOLECULAR MECHANISMS OF FEAR LEARNING AND MEMORY. Cell, 147, 509-524.

Jones, E.G. (1998) Viewpoint: the core and matrix of thalamic organization. Neuroscience, $85,331-45$.

Kim, S., Mátyás, F., Lee, S., Acsády, L., \& Shin, H.-S. (2012) Lateralization of observational fear learning at the cortical but not thalamic level in mice. Proc. Natl. Acad. Sci. U. S. A., $109,15497-15501$. 
Kita, H. \& Kitai, S.T. (1990) Amygdaloid projections to the frontal cortex and the striatum in the rat. J. Comp. Neurol., 298, 40-49.

Krettek, J.E. \& Price, J.L. (1977) Projections from the amygdaloid complex to the cerebral cortex and thalamus in the rat and cat. J. Comp. Neurol., 172, 687-722.

Krout, K.E., Belzer, R.E., \& Loewy, A.D. (2002) Brainstem projections to midline and intralaminar thalamic nuclei of the rat. J Comp Neurol, 448, 53-101.

Kuroda, M. \& Price, J.L. (1991) Synaptic organization of projections from basal forebrain structures to the mediodorsal thalamic nucleus of the rat. J. Comp. Neurol., 303, 513533.

Lavallee, P., Urbain, N., Dufresne, C., Bokor, H., Acsady, L., \& Deschenes, M. (2005) Feedforward inhibitory control of sensory information in higher-order thalamic nuclei. $J$ Neurosci, 25, 7489-7498.

LeDoux, J., Farb, C., \& DA, R. (1990) Topographic Organization Project to the Amygdala of Neurons in the Acoustic Thalamus That. J. Neurosci., 10, 1043.

Lee, S., Ahmed, T., Lee, S., Kim, H., Choi, S., Kim, D.-S., Kim, S.J., Cho, J., \& Shin, H.-S. (2012) Bidirectional modulation of fear extinction by mediodorsal thalamic firing in mice. Nat. Neurosci., 15, 308-314.

Li, S. \& Kirouac, G.J. (2008) Projections from the paraventricular nucleus of the thalamus to the forebrain, with special emphasis on the extended amygdala. J. Comp. Neurol., 506, 263-287.

Marek, R., Strobel, C., Bredy, T.W., \& Sah, P. (2013) The amygdala and medial prefrontal cortex: partners in the fear circuit. J. Physiol., 591, 2381-2391.

McDonald, A.J. (1991) Organization of amygdaloid projections to the prefrontal cortex and associated striatum in the rat. Neuroscience, 44, 1-14.

McDonald, A.J. (1998) Cortical pathways to the mammalian amygdala. Prog. Neurobiol., 55, $257-332$.

Mcdonald, A.J., Mascagni, F., \& Guo, L. (1996) Projections of the medial and lateral prefrontal cortices to the amygdala: a Phaseolus vulgaris leucoagglutinin study in the rat. Neuroscience, 71, 55-75.

Ottersen, O.P. (1982) Connections of the amygdala of the rat. IV: Corticoamygdaloid and intraamygdaloid connections as studied with axonal transport of horseradish peroxidase. J. Comp. Neurol., 205, 30-48.

Ottersen, O.P. \& Ben-Ari, Y. (1979) Afferent connections to the amygdaloid complex of the rat and cat. I. Projections from the thalamus. J. Comp. Neurol., 187, 401-424. 
Pape, H.-C. \& Pare, D. (2010) Plastic synaptic networks of the amygdala for the acquisition, expression, and extinction of conditioned fear. Physiol. Rev., 90, 419-463.

Paxinos, G. \& Franklin, K. (2013) The Mouse Brain in Stereotaxix Coordinates, 4th Editio. edn. Academic Press.

Ray, J.P. \& Price, J.L. (1992) The organization of the thalamocortical connections of the mediodorsal thalamic nucleus in the rat, related to the ventral forebrain-prefrontal cortex topography. J. Comp. Neurol., 323, 167-197.

Ray, J.P., Russchen, F.T., Fuller, T.A., \& Price, J.L. (1992) Sources of presumptive glutamatergic/aspartatergic afferents to the mediodorsal nucleus of the thalamus in the rat. J. Comp. Neurol., 320, 435-456.

Reardon, F. \& Mitrofanis, J. (2000) Organisation of the amygdalo-thalamic pathways in rats. Anat. Embryol. (Berl)., 201, 75-84.

Sah, P., Faber, E.S.L., Lopez De Armentia, M., \& Power, J. (2003) The amygdaloid complex: anatomy and physiology. Physiol. Rev., 83, 803-834.

Sherman, S.M. \& Guillery, R.W. (1998) On the actions that one nerve cell can have on another: distinguishing \&quot;drivers\&quot; from \&quot;modulators\&quot. Proc Natl Acad Sci U S A, 95, 7121-6.

Shinonaga, Y., Takada, M., \& Mizuno, N. (1994) Topographic organization of collateral projections from the basolateral amygdaloid nucleus to both the prefrontal cortex and nucleus accumbens in the rat. Neuroscience, 58, 389-397.

Slézia, A., Hangya, B., Ulbert, I., \& Acsády, L. (2011) Phase advancement and nucleusspecific timing of thalamocortical activity during slow cortical oscillation. J. Neurosci., 31, 607-617.

Sripanidkulchai, K., Sripanidkulchai, B., \& Wyss, J.M. (1984) The cortical projection of the basolateral amygdaloid nucleus in the rat: a retrograde fluorescent dye study. J. Comp. Neurol., 229, 419-431.

Su, H.S. \& Bentivoglio, M. (1990) Thalamic midline cell populations projecting to the nucleus accumbens, amygdala, and hippocampus in the rat. J. Comp. Neurol., 297, 582593.

Van De Werd, H.J.J.M., Rajkowska, G., Evers, P., \& Uylings, H.B.M. (2010) Cytoarchitectonic and chemoarchitectonic characterization of the prefrontal cortical areas in the mouse. Brain Struct. Funct., 214, 339-353.

Vertes, R.P. (2004) Differential projections of the infralimbic and prelimbic cortex in the rat. Synapse, 51, 32-58.

Vogt, B.A., Vogt, L., \& Farber, N. (2004) Cingulate cortex and models of disease. In Paxinos, G. (ed), The Rat Nervous System, 3rd editio. edn. Elsevier Academic Press, pp. 704-760. 
Wang, C.-C. \& Shyu, B.-C. (2004) Differential projections from the mediodorsal and centrolateral thalamic nuclei to the frontal cortex in rats. Brain Res., 995, 226-235. 


\section{FIGURES AND FIGURE LEGENDS}

Figure 1. Parcellation of medial prefrontal cortex using the combination neurochemical and connectional criteria. Borders (arrows) of cingulate $(\mathrm{Cg})$, pre- (PrL) and infralimbic (IL) cortices as defined on low power light micrographs using parvalbumin (Parv) immunoreactivity (A), antero- and retrograde tracing from the amygdala (B-D) and anterograde tracing from the midline thalamic nuclei (E) at two coronal levels (top raw rostral, bottom row, caudal). PrL is defined by a sharp drop in the intensity of superficial parvalbumin immunoreactivity and amygdalar input, a shift of cortico-amygdalar cells to deeper layers and a prominent input from the midline thalamus. The amygdalar tracing was performed by co-injecting the antero- and retrograde tracers. Note that the position of the dorsal border changes very little relative to the dorsal surface of the brain between the two antero-posterior levels. M2, secondary motor cortex.

Figure 2. Delineation of mediodorsal and midline thalamic nuclei using vGLUT2 immunostaining. A-E) Borders of medidorsal (MD) and midline thalamic nuclei as defined on low power light micrographs displaying vGLUT2 immunostaining at five coronal levels (right column). The border is the result of a dense glutamatergic input from the brainstem to the midline nuclei. Left column; the same images with an overlay of nuclear borders. Asterisks in the first two levels indicate the transition zone between the midline thalamus and MD, where the border, as defined by vGLUT2 immunostaining, is less conspicuous. AV, anteroventral n., CL, centrolateral n; CM, centromedial thalamic n.; Hb, habenula; IAD, interanterodorsal n.; IAM, interanteromedial n.; IMD, intermediodorsal thalamic n.; PC, paracentral n.; PV, paraventricular n.; Pt, paratenial n. 
Figure 3. Distribution of corticothalamic neurons following retrograde tracer injection into the mediodorsal thalamic nucleus. A-C) Low power light micrographs of corticothalamic cells projecting to MD from the prefrontal cortex at three coronal levels arranged from anterior to posterior. Note that the neurons located close to the medial wall in B belong to the orbital (VO/LO) and cingulate ( $\mathrm{Cg}$ ) cortices, and not to prelimbic (PrL) or infralimbic (IL) cortices, as can be seen on the higher power image (E). D) Injection site in the rostral part of MD double immunostained for vGLUT2 and Cholera Toxin-B subunit (CTB). E,F) Double immunostainings for Parv and CTB made from the boxed area to differentiate the deep layers of orbital and prelimbic cortices. White arrows and lines indicate the border between $\mathrm{Cg}$ and PrL cortices based on Parv-immunostaining.

Figure 4. Corticothalamic fibres arising from cingulate, prelimbic/infralimbic and insular cortices innervate distinct sectors in the medial thalamus. Distribution of corticothalamic axons following the injection of anterograde tracer to cingulate $(\mathrm{A} ; \mathrm{Cg})$, prelimbic (D; PrL) and insular (G; AI) cortical regions. A and D are double immunostained for parvalbumin, the blue staining is DAPI in G. All thalamic sections are double immunostained for vGLUT2 to label the boundaries between MD and the midline nuclei and are shown at two coronal levels. The border between Cg and PrL cortices is indicated by white arrows and lines in A and D. Note dense but non-overlapping innervation of lateral and medial MD in case of cingulate (BC) and insular (H-I) injections, respectively. In case of the prelimbic injection most MD axons are localized to the rostral and medial transition zone of MD (E) and taper off more caudally (F). IMD, intermediosorsal thalamic n.; PV, paraventricularis thalamic n.

Figure 5. Distribution of thalamocortical neurons projecting to cingulate, prelimbic, and insular cortices. Complementing the anterograde tracing, the distribution of thalamocortical 
cells following the injection of retrograde tracers $(\mathrm{CTB})$ in cingulate $(\mathrm{A} ; \mathrm{Cg})$, prelimbic $(\mathrm{D}$; PrL) and insular (G; AI) cortices closely matches that of the innervation pattern of corticothalamic fibres (compare to Fig. 4). Thalamocortical cells projecting to cingulate and insular cortex are localized in lateral (B-C) and medial (H-I) MD, respectively, whereas those innervating prelimbic cortex are situated in the transition zone. Counterstainings are the same as in Fig. 4. IMD, intermediosorsal thalamic n.; PV, paraventricularis thalamic n.

Figure 6. Lack of major input from the basolateral amygdala to the mediodorsal nucleus retrograde tracing. A) Low power image of the injection site in MD and the resulting retrograde labelling in the ventral endopiriform nucleus (vEN). B) Higher power image of the boxed area. Inset shows the retrogradely filled vEN neurons. C) Retrogradely labelled cells at a more caudal level in the same animal. Note the paucity of cell labelling in BLA and its presence in VEn. CeA, central amygdaloid n.; LOT, lateral n. of the olfactory tract; MeA, medial amygdaloid $n$.

Figure 7. Lack of major input from the basolateral amygdala to the mediodorsal nucleus anterograde tracing. A-C) Injection in BLA avoiding its lateral margin results in no fibre labelling in MD. D-F) Injection in lateral BLA and the neighbouring ventral endopiriform nucleus (VEn) labels fibres in the medial part of MD, shown in low power fluorescent micrographs double immunostained for vGLUT2. G-H) High power confocal images show the anterogradely labelled axons which are vGLUT1-negative (G) but positive for vGLUT2 (H). CeA, central amygdaloid nuclei; CL, centrolateral thalamic n; CM, centromedial thalamic n.; IMD, intermediodorsal thalamic n.; LA, lateral amygdala; PC, paracentral thalamic n.; PV, paraventricular thalamic n. 
Figure 8. Distribution of thalamic neurons projecting the basolateral amygdala. A) Low power image of the injection site in the amygdala. B-D) Distribution of retrogradely labelled cells in the medial thalamus at three antero-posterior levels double immunostained for vGLUT2. Note that the vast majority of the neurons are localized within the midline nuclei and the medial transition zone. CeA, central amygdaloid nuclei; CL, centrolateral thalamic n; CM, centromedial thalamic n.; IMD, intermediodorsal thalamic n.; PC, paracentral thalamic n.; PV, paraventricular thalamic $n$.

Figure 9. Distribution of cortical cells projecting to the basolateral amygdala. A-C) Low power light micrographs of cortical cells projecting to BLA from the prefrontal cortex at three coronal levels arranged from anterior to posterior. For the injection site see Fig. 8. D-F) Higher power images of the boxed regions displaying projecting cells in the cingulate $(\mathrm{Cg}$; $\mathrm{D}$ E) and insular (AI; F) cortical regions. Note that majority of the cortico-amygdalar cells are located in superficial layers of cingulate cortex but involves deeper layers as well in prelimbic and insular areas. Black arrows and line mark the ventral border of $\mathrm{Cg}$ in E. IL, infralimbic cortex; M1 and M2, primary and secondary motor cortex; PrL, Prelimbic cortex; VO/LO, ventral and lateral orbital cortices.

Figure 10. Connections of basolateral amygdala with cingulate, prelimbic and insular cortical regions. A-F) Distribution of cortico-amygdaloid fibres in the BLA, following the injection of anterograde tracer into the cingulate (A-B), prelimbic (C-D) and insular (E-F) cortices. G-L) Distribution of amygdalo-cortical cells in BLA after retrograde tracer injections into the cingulate $(\mathrm{G}-\mathrm{H})$, prelimbic cortices (I-J) and insular (K-L). The same animals were used for anterograde tracing as in Fig. 4 and for retrograde experiments as in Fig. 5. All sections are immunostained for vGLUT2 to label nuclear boundaries and are shown at two coronal levels. 
Note close correspondence of the distribution of cortico-amydaloid fibres and amygdalocortical cells. CeA, central amygdala; LA, lateral amygdala.

Figure 11. Amygdalo-cortical fibres have a wider distribution than cortico-amygdaloid cells. A-C) Low power fluorescent light micrographs of amygdalo-cortical fibres projecting to the prefrontal cortex at three coronal levels arranged from anterior to posterior and counterstained with DAPI. Note wider distribution of fibres compared to cortical regions innervating amygdala (Fig. 9) and innervation of cortical regions (LO, VO, M2) which project to MD (compare to Fig. 3). D) Injection site in the amygdala. E-F) Higher power images showing the labelled fibres along the cingulate $(\mathrm{Cg}) /$ prelimbic border $(\operatorname{PrL} ; \mathrm{E})$ and in the insulate cortex (AI). White arrows and line label the $\mathrm{Cg} / \mathrm{PrL}$ border. IL, infralimbic cortex; M1 and M2, primary and secondary motor cortex. VO/LO, ventral and lateral orbital cortex.

Figure 12) Schematic representation of the connectivity between basolateral amygdala, prefrontal cortex and the mediodorsal nucleus of the thalamus. A) From the six possible connections among the PL/IL cortex, BLA and MD five have been documented in rats, suggesting a closed triangular loop between the three regions. B) In our tracing study only the reciprocal connection between PL/IL and BLA proved to be sufficiently strong to play major physiological roles in mice. C) BLA and MD, however, can interact via their mutual, reciprocal connections with insular cortex (AI) and the cingulate/secondary motor cortices (Cg/M2) border region. In addition, amygdala can influence MD activity (gray arrow in C) via an unidirectional pathway to ventral and lateral orbital cortices (VO/LO) which in turn are reciprocally coupled to $\mathrm{MD}$. 


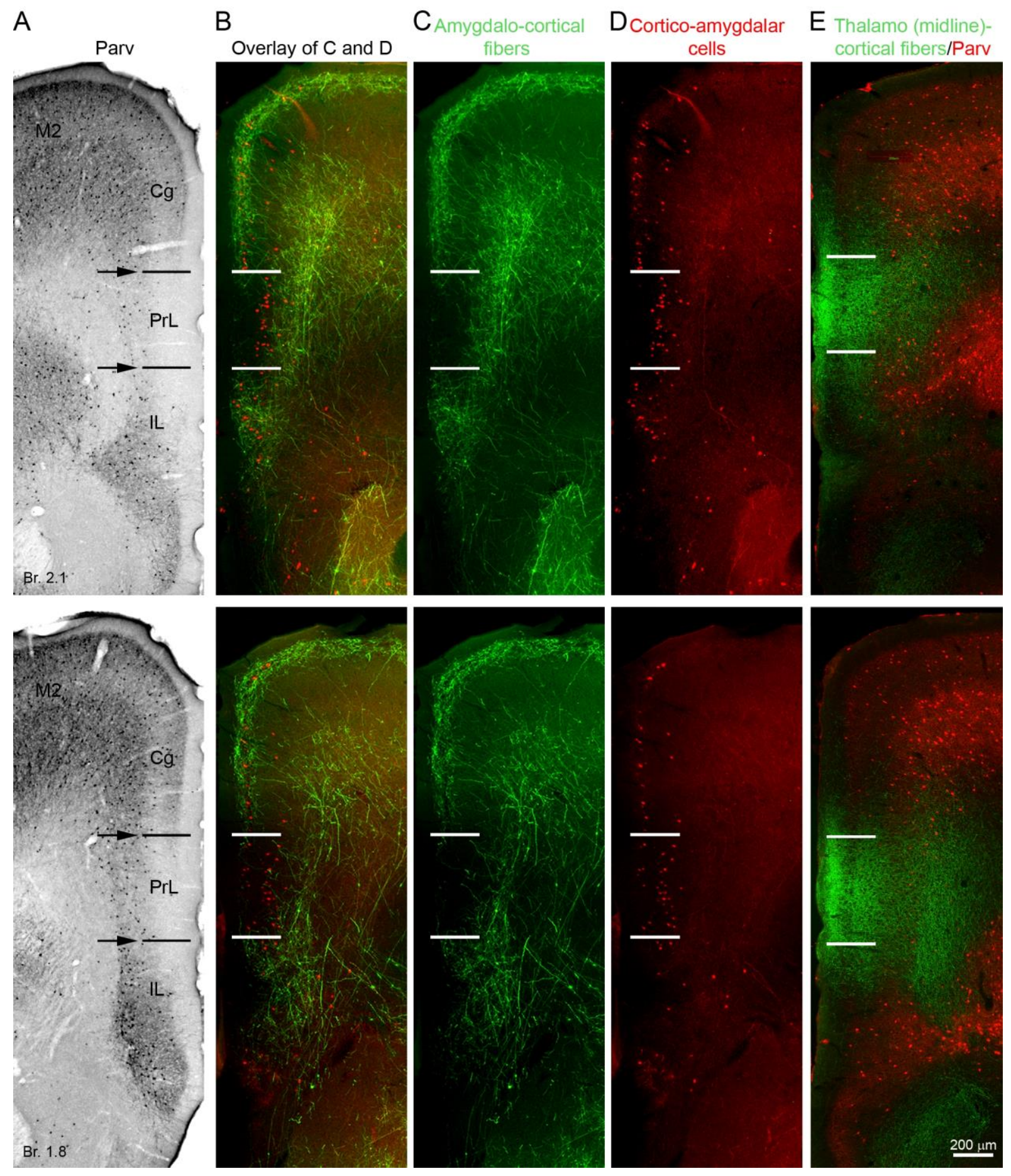

Figure 1. 

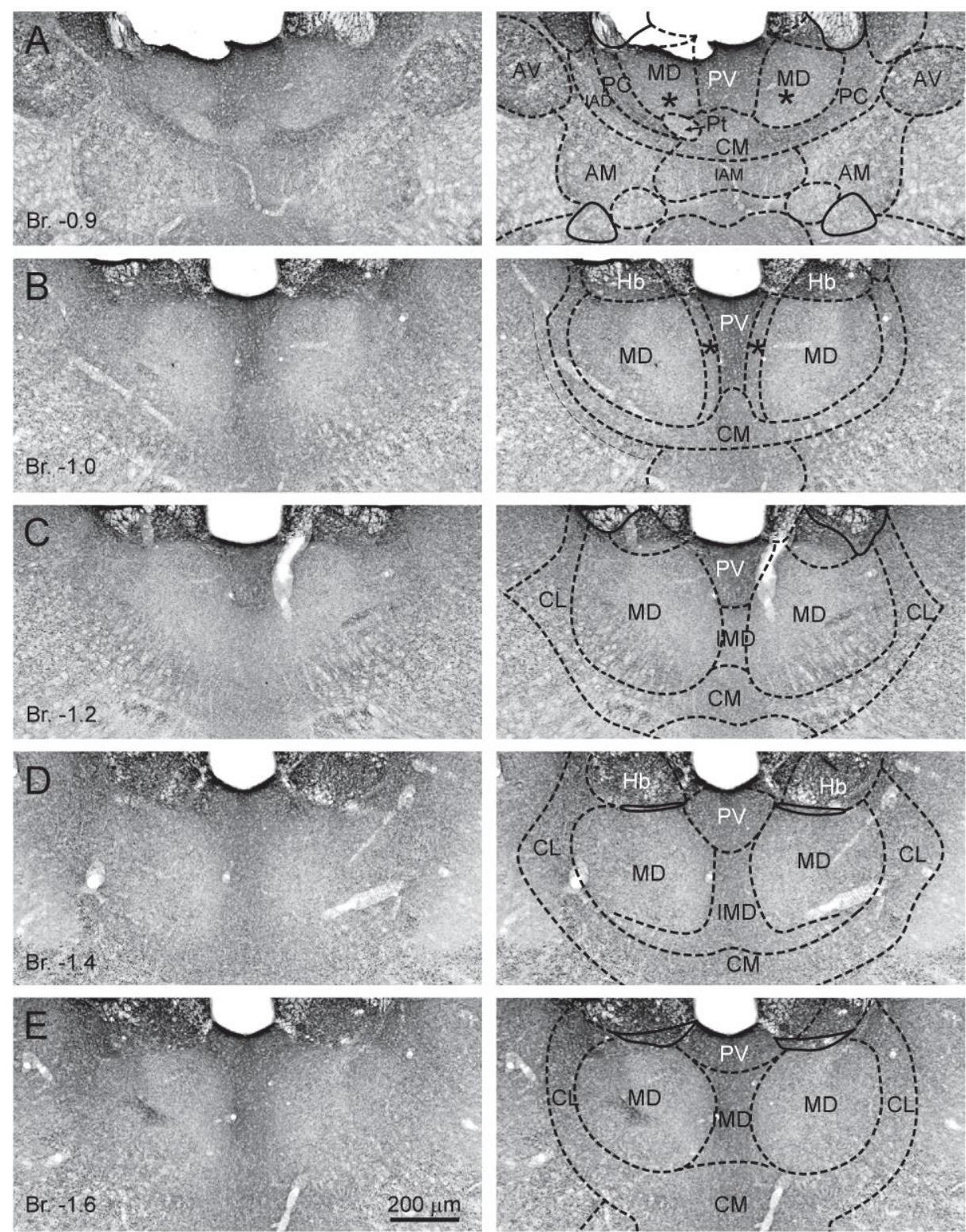

Figure 2. 

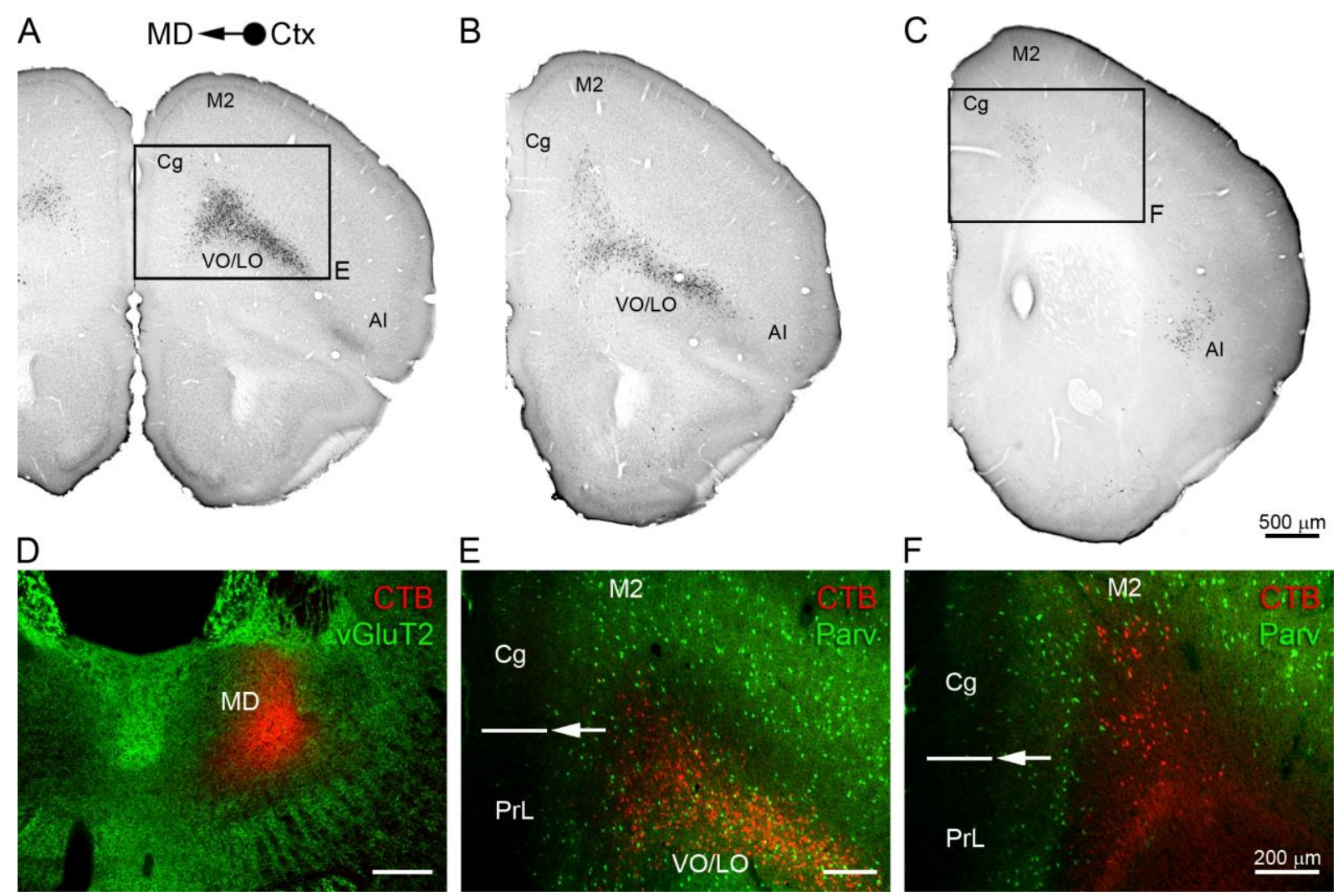

Figure 3. 

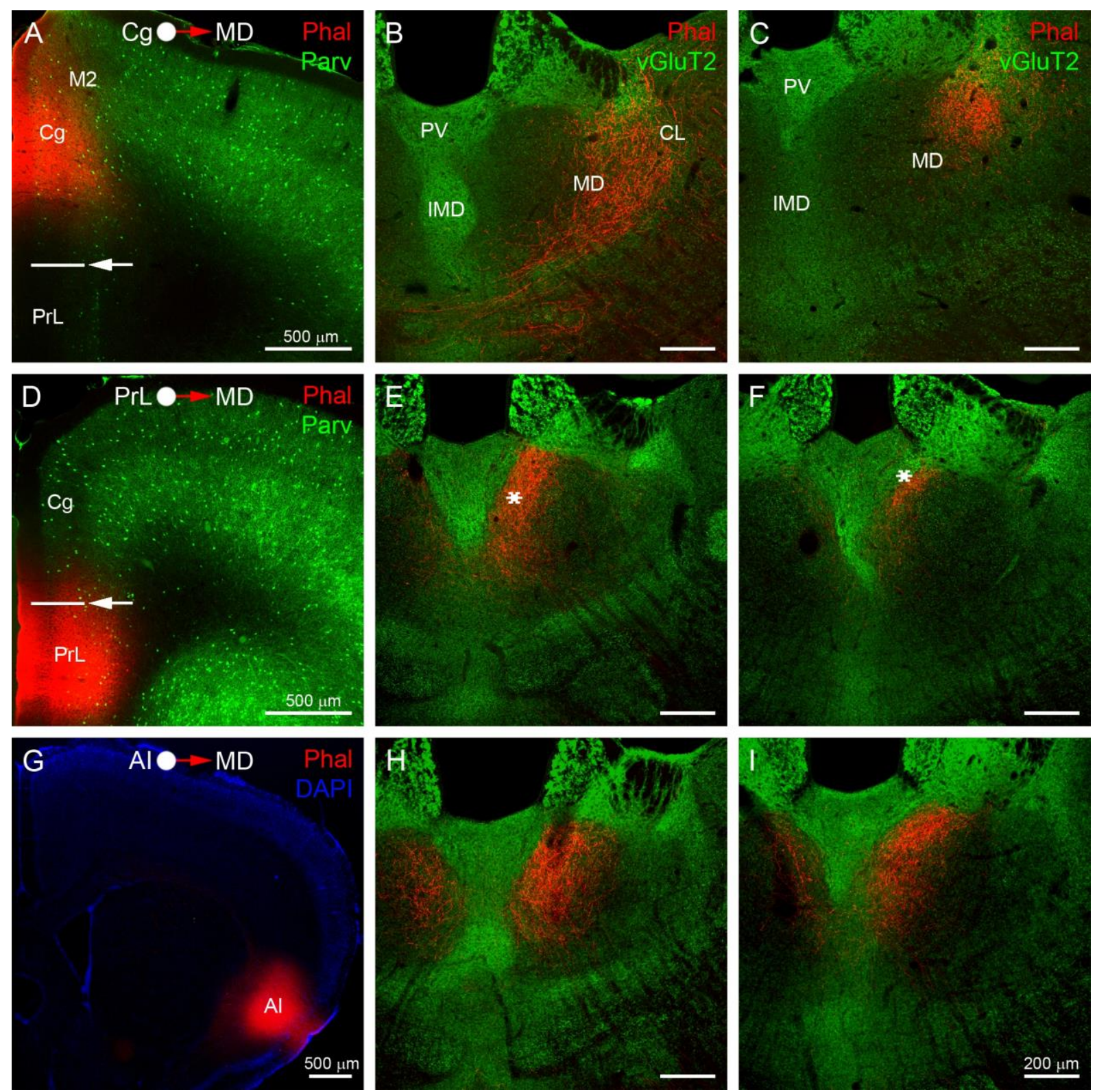

Figure 4. 

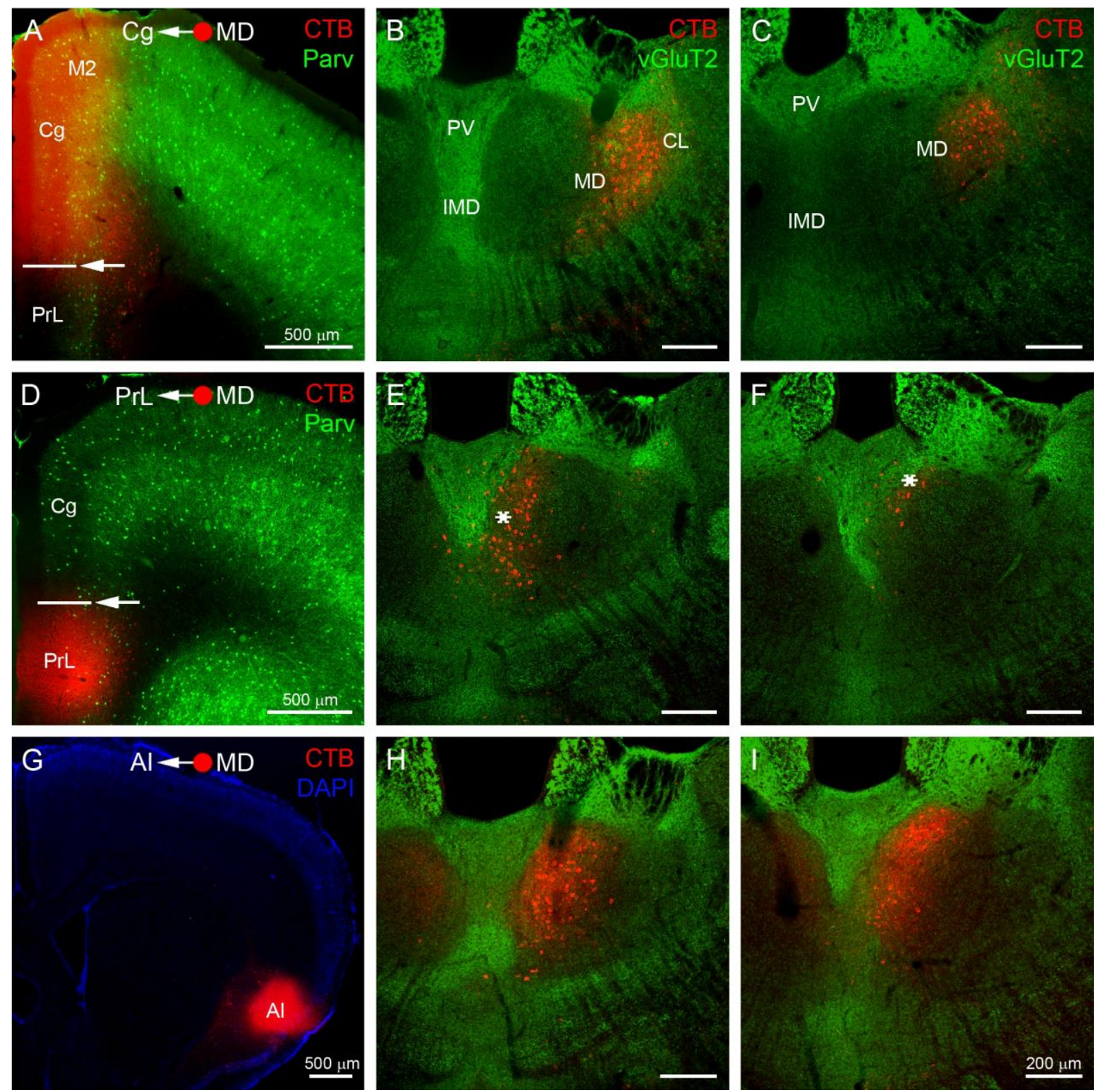

Figure 5.
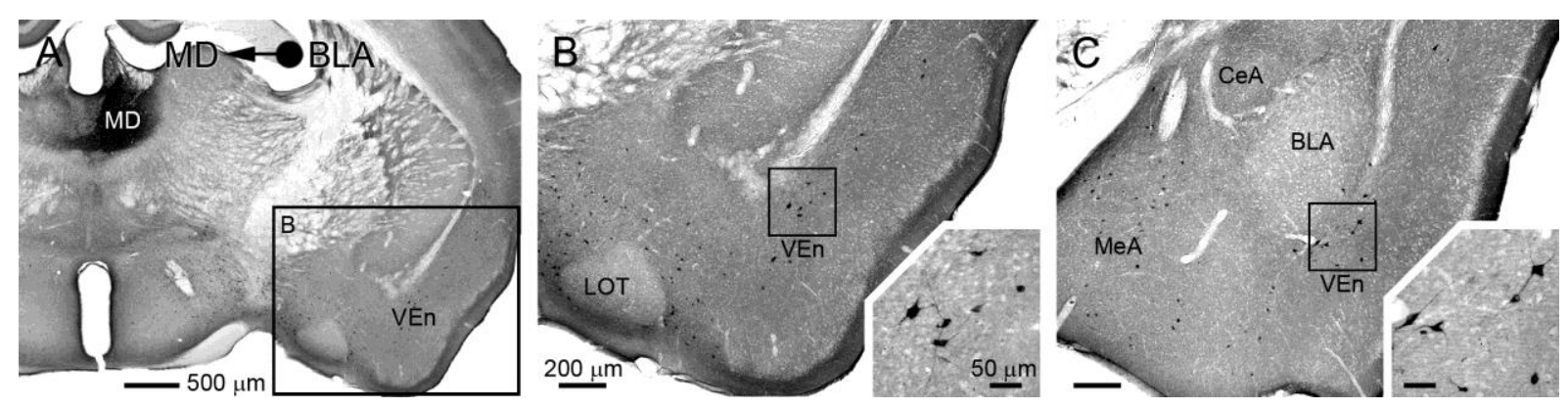

Figure 6. 

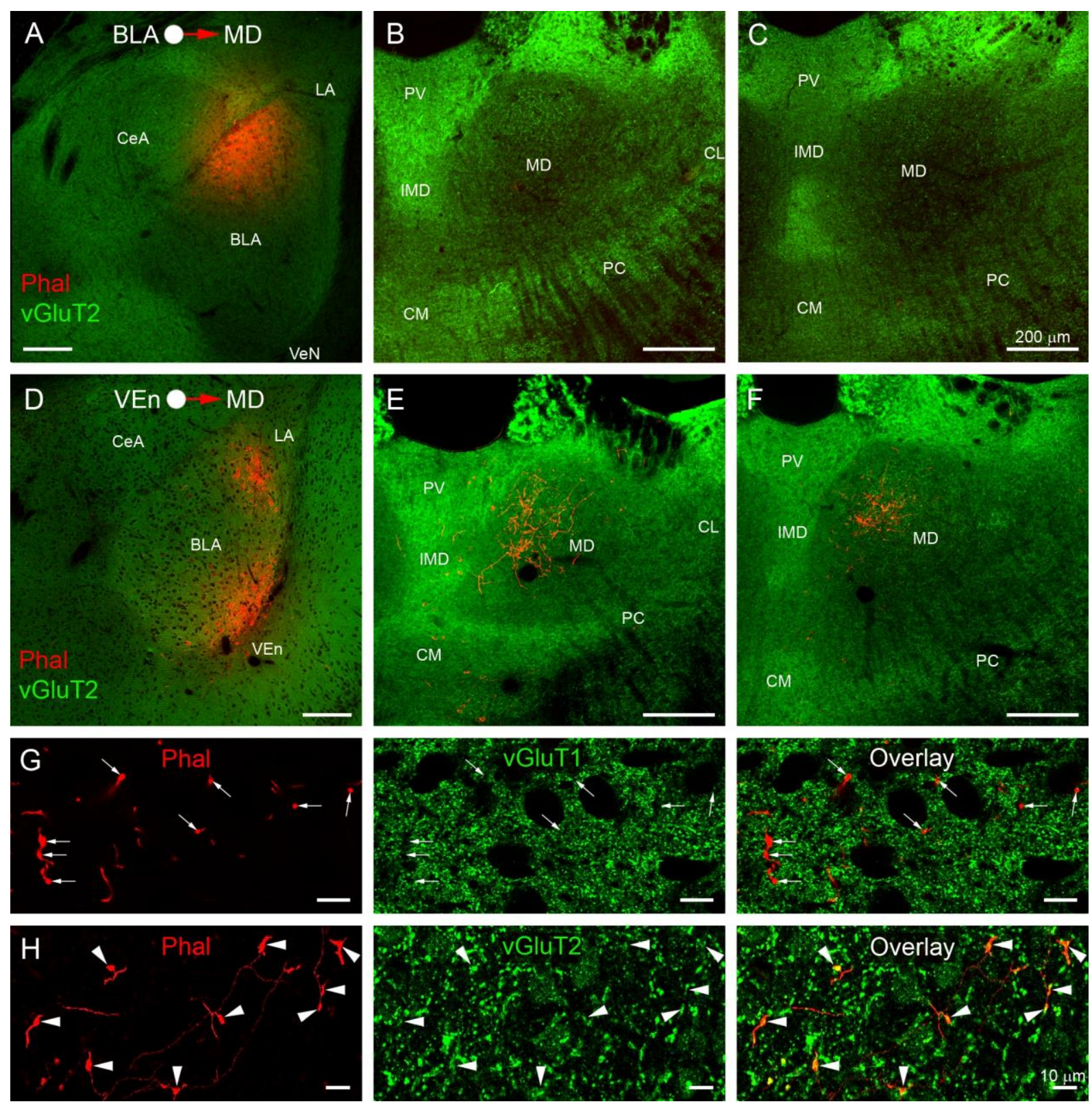

Figure 7.
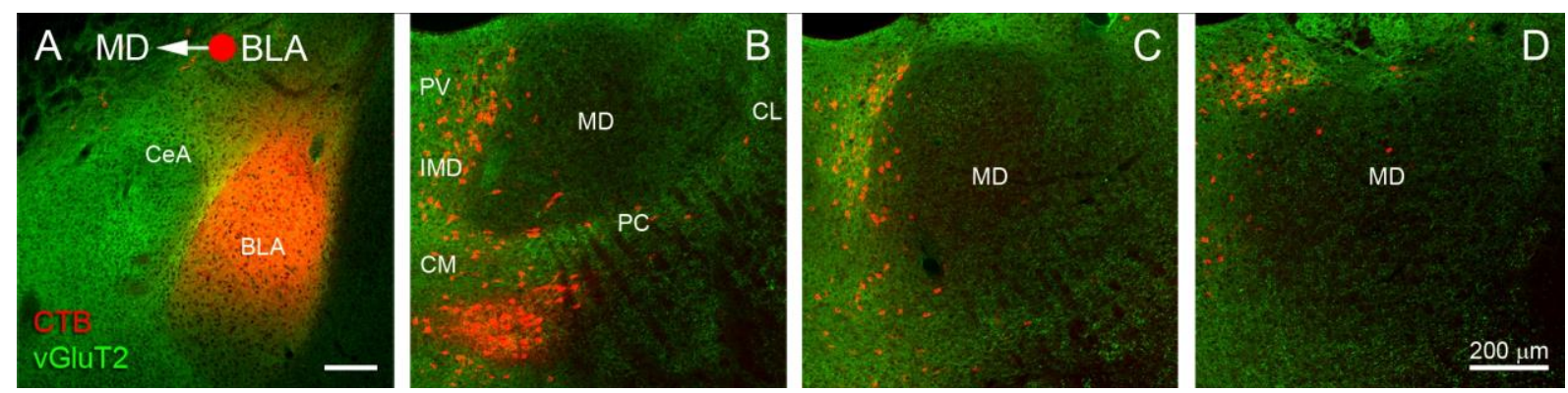

Figure 8. 

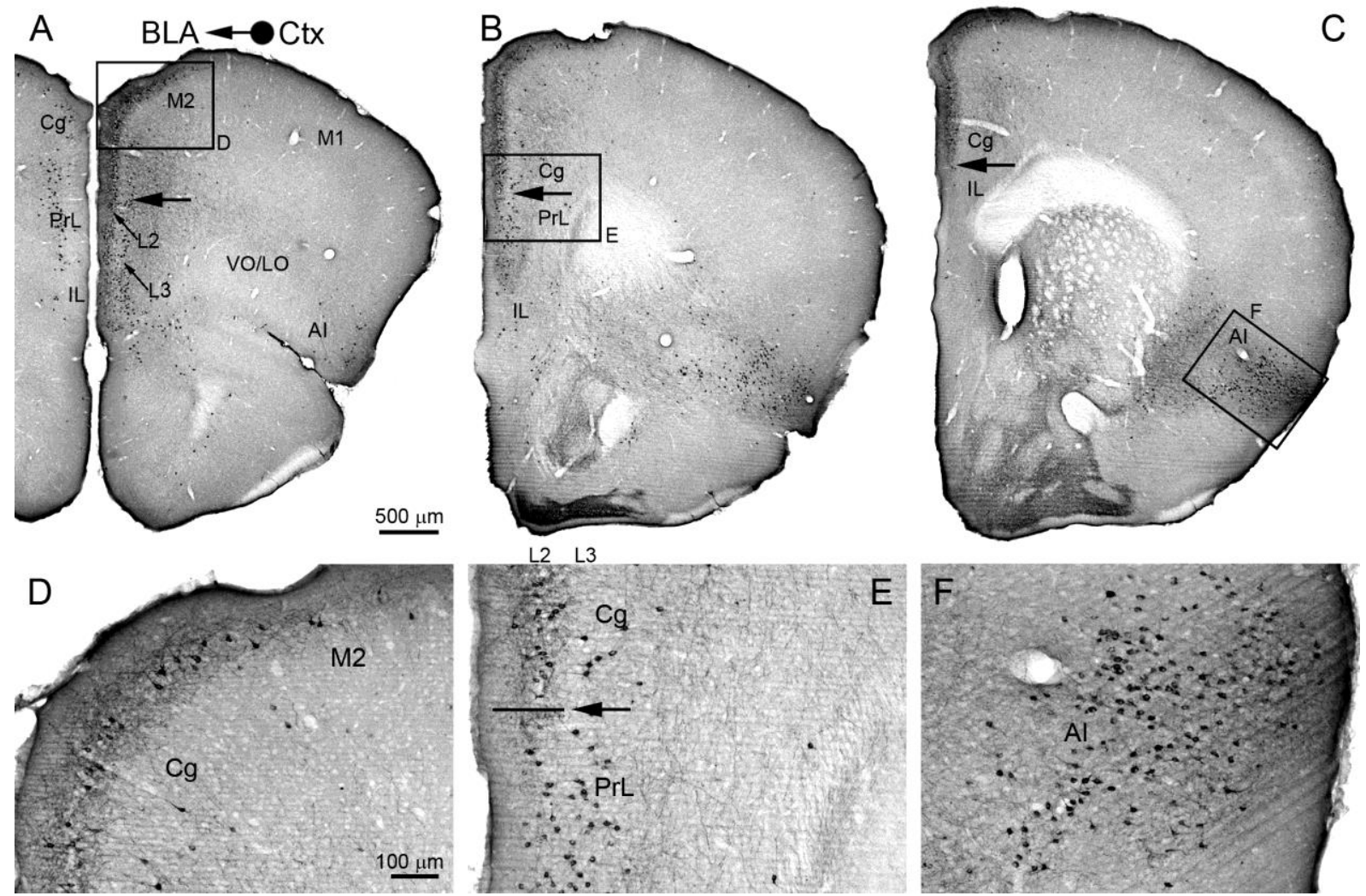

Figure 9.
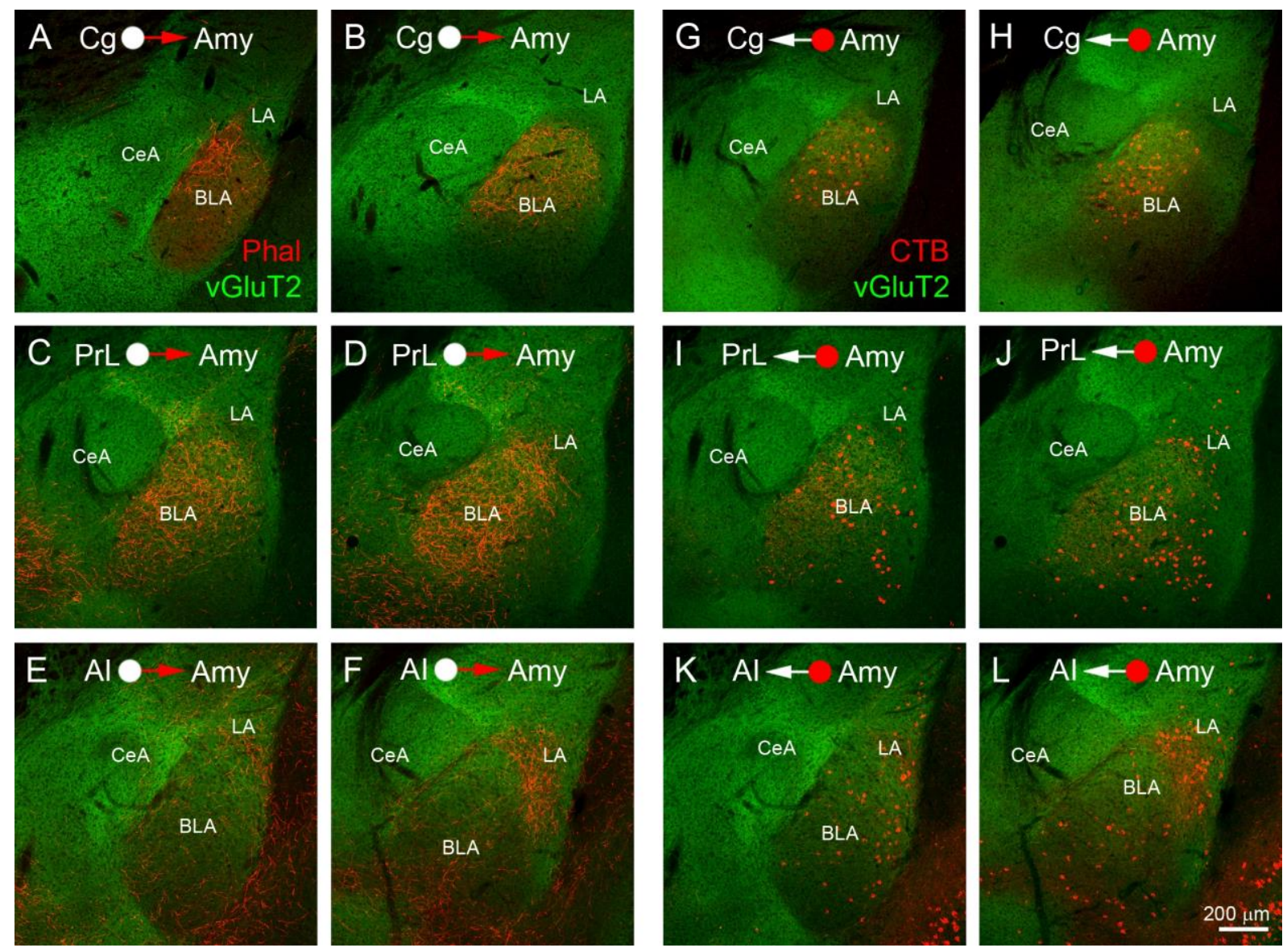

Figure 10. 

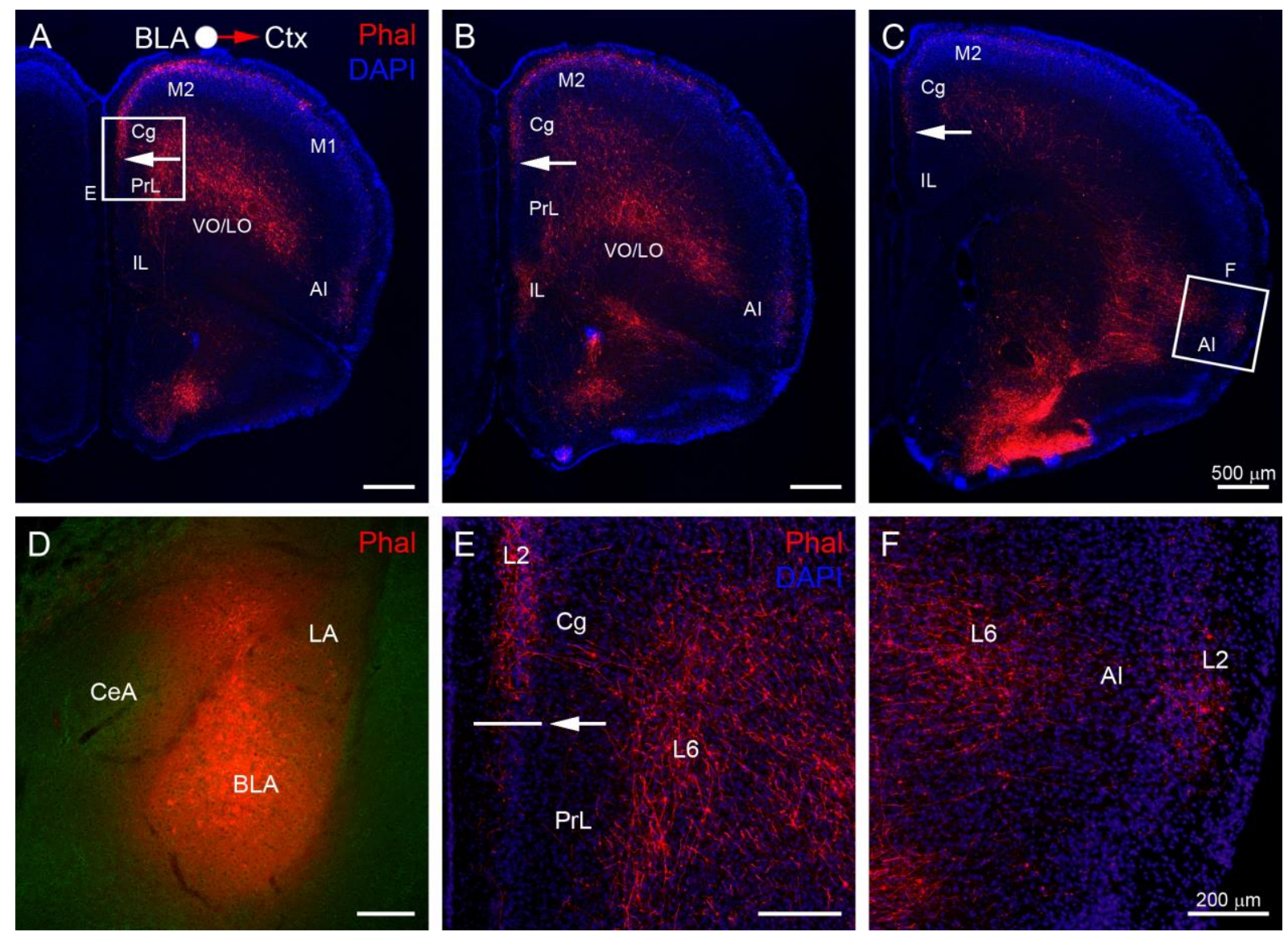

Figure 11.

A Published data

B Current data
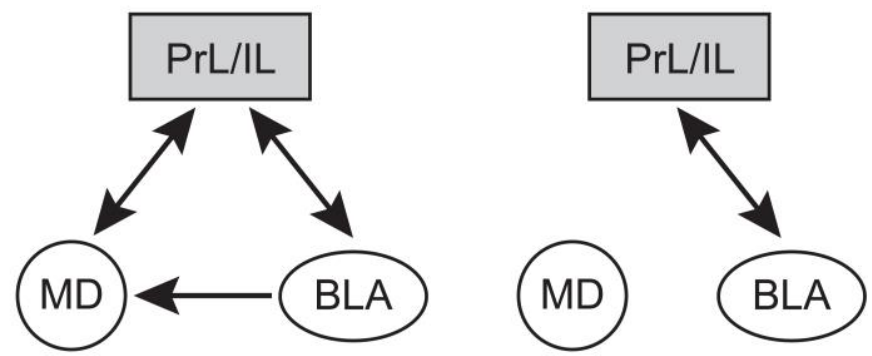

C Other connectivites

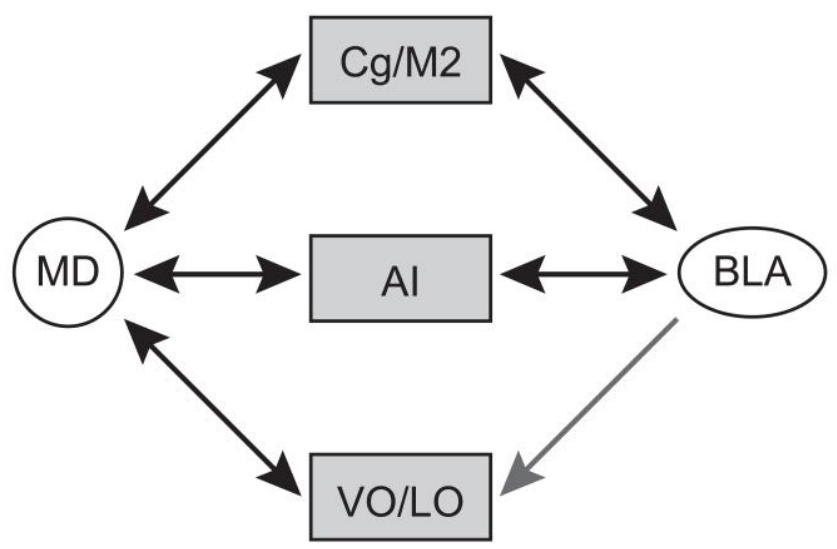

Figure 12. 